doi: $10.17951 /$ a.2016.70.1.47

A N N A LES

UNIVERSITATIS MARIAE CURIE-SKŁODOWSKA

L UBLIN - POLONIA

VOL. LXX, NO. 1, 2016

SECTIO A

$47-74$

KRZYSZTOF PILORZ

\title{
A kinetic equation for repulsive coalescing random jumps in continuum
}

\begin{abstract}
A continuum individual-based model of hopping and coalescing particles is introduced and studied. Its microscopic dynamics are described by a hierarchy of evolution equations obtained in the paper. Then the passage from the micro- to mesoscopic dynamics is performed by means of a Vlasovtype scaling. The existence and uniqueness of solutions of the corresponding kinetic equation are proved.
\end{abstract}

1. Introduction. In this paper, we introduce and study the dynamics of an infinite system of particles located in $\mathbb{R}^{d}$, which jump and merge (coalesce). Both jumping and coalescing are repulsive. The proposed model is individual based, which means that the description of its Markov dynamics is performed in terms of random changes of states of individual particles. In the proposed model, such changes include: (a) the particle located at a given $x \in \mathbb{R}^{d}$ changes its position to $y \in \mathbb{R}^{d}$ (jumping); (b) two particles, located at $x \in \mathbb{R}^{d}$ and $y \in \mathbb{R}^{d}$, merge into a single particle located at $z \in \mathbb{R}^{d}$ (coalescing). The rates of these events depend on the configuration of all particles. Similar single type population models are used to describe predation in ecology, see, e.g., [6]. The model proposed can be viewed as an extension of the Kawasaki model with repulsion studied in [5] where only random jumps with repulsion are taken into account. To the best of our

2010 Mathematics Subject Classification. 60K35, 35Q83, 82C22.

Key words and phrases. Coalescence, coagulation, hopping particles, individual-based model, configuration spaces, infinite particle system, microscopic dynamics, Vlasov scaling, kinetic equation. 
knowledge, the microscopic modeling of this kind of merging is performed here for the first time. Previous theories, see, e.g., [1, 2, 3, 4, 11, 13, 14], deal with the particle densities and hence do not take into account the corpuscular structure of the system or, see, e.g., $[15,16]$, while introducing individual-based models, describe only finite systems with coalescence between two particles dependent on characteristics of the particles involved and the mass of entire population. Interactions between particles are not taken into account. The most important aspects of the model proposed in this paper are: (a) the coalescence rate depends on the neighbourhood of the coalescing particles; (b) infinite systems of particles are described. However, the particle mass is not taken into account, which is supposed to be done in an extension of the present model.

The state space of the system considered is the configuration space, that is, the set of all locally finite subsets of $\mathbb{R}^{d}$

$$
\Gamma=\Gamma\left(\mathbb{R}^{d}\right)=\left\{\gamma \subset \mathbb{R}^{d}: \gamma \cap \Lambda \text { is finite for every compact } \Lambda \subset \mathbb{R}^{d}\right\} .
$$

It can be given a measurability structure. Take

$$
\mathcal{F}=\sigma\left(\Gamma_{n, \Lambda}: n \in \mathbb{N}_{0}, \Lambda \subset \mathbb{R}^{d} \text { - compact }\right),
$$

that is the smallest $\sigma$-field generated by the cylinders

$$
\Gamma_{n, \Lambda}=\{\gamma \in \Gamma:|\gamma \cap \Lambda|=n\}
$$

This allows one to consider probability measures on standard Borel space $(\Gamma, \mathcal{F})$ as states of the system. To characterize them one uses observables, which are appropriate functions $F: \Gamma \rightarrow \mathbb{R}$. For an observable $F$ and a state $\mu$, the number

$$
\int_{\Gamma} F d \mu
$$

is the $\mu$-expected value of $F$. Then the evolution of states $\mu_{0} \mapsto \mu_{t}$ can be described via the dual evolution $F_{0} \mapsto F_{t}$ based on the following duality relation

$$
\int_{\Gamma} F_{0} d \mu_{t}=\int_{\Gamma} F_{t} d \mu_{0}, \quad t>0
$$

The evolution of observables is obtained in turn from the Kolmogorov equation

$$
\frac{d}{d t} F_{t}=L F_{t},\left.\quad F_{t}\right|_{t=0}=F_{0},
$$


in which the "operator" $L$ characterizes the model, see $[7,8,9,10]$ for more details. In the proposed model, it has the following form

$$
\begin{aligned}
L F(\gamma)= & \sum_{\{x, y\} \subset \gamma} \int_{\mathbb{R}^{d}} \tilde{c}_{1}(x, y ; z ; \gamma)(F(\gamma \backslash\{x, y\} \cup z)-F(\gamma)) d z \\
& +\sum_{x \in \gamma} \int_{\mathbb{R}^{d}} \tilde{c}_{2}(x ; y ; \gamma)(F(\gamma \backslash x \cup y)-F(\gamma)) d y .
\end{aligned}
$$

The first term of $L$ describes the coalescence occurring with intensity $\tilde{c}_{1}(x, y ; z ; \gamma)$. The particles located at $x$ and $y$ merge into a new particle located at a point $z$. The second term describes the jump of the particle located at $x$ to a point $y$ with intensity $\tilde{c}_{2}(x ; y ; \gamma)$. The model with $L$ consisting of the second term only is the Kawasaki model studied in [5]. The kernels $\tilde{c}_{1}$ and $\tilde{c}_{2}$ take into account also the influence of the whole configuration, which is supposed to be repulsive, see (3.2) below. Note that $L$ is linear and independent of time and hence we deal with Markov dynamics.

In the present research, we follow the statistical approach, see, e.g., [5, $7,8,9,10]$, in which the dynamics of the model are described by means of that of the corresponding correlation functions obtained from the following Cauchy problem

$$
\frac{d}{d t} k_{t}=L^{\Delta} k_{t}, \quad k_{t=0}=k_{0},
$$

in which the "operator" $L^{\Delta}$ is related to $L$ in a certain way. In Section 3 , we calculate $L^{\Delta}$ following the scheme developed in [10]. Usually, equations for $k_{t}$ are studied in scales of the corresponding Banach spaces. However, as the structure of $L^{\Delta}$ obtained below is too complicated, in this work we do not study this equation, which is supposed to be done in a separate work. Instead, in Section 4 we consider a simplified version obtained by means of a Vlasov-type scaling procedure developed in, e.g., [8], which is equivalent to passing to the so-called mesoscopic description. In particular, we informally obtain the kinetic equation and study its local solutions in an appropriate Banach space, showing their existence and uniqueness. In the next section, we introduce necessary notions and technical tools.

2. Basic notions and tools. In this section we introduce basic notions and tools used for proving the results in the following sections. We give only a short description with references to the corresponding sources.

Note that each element $\gamma \in \Gamma$ is at most countable without finite limiting points. $\Gamma$ is endowed with the vague topology, which is the weakest topology that makes continuous the mappings

$$
\gamma \rightarrow \sum_{x \in \gamma} f(x)
$$


for all continuous compactly supported functions $f: \mathbb{R}^{d} \rightarrow \mathbb{R}$. For the general discussion on configuration spaces we recommend $[7,9]$.

One can also consider the space of all finite configurations

$$
\Gamma_{0}=\Gamma_{0}\left(\mathbb{R}^{d}\right)=\{\eta \in \Gamma: \eta \text { is finite }\}
$$

By $B_{b s}\left(\Gamma_{0}\right)$ we denote the set of all bounded measurable functions $G$ : $\Gamma_{0} \rightarrow \mathbb{R}$ such that for some $N \in \mathbb{N}_{0}$ and compact $\Lambda \subset \mathbb{R}^{d}$, we have $G^{(n)}\left(x_{1}, \ldots, x_{n}\right)=0$, whenever $n \geq N$ or one of the arguments $x_{i} \notin \Lambda$. Then the $K$-transform is defined as follows. For any $G \in B_{b s}\left(\Gamma_{0}\right), K G: \Gamma \rightarrow \mathbb{R}$ is

$$
(K G)(\gamma)=\sum_{\eta \Subset \gamma} G(\eta),
$$

where $\eta \Subset \gamma$ means that $\eta$ is a finite sub-configuration of $\gamma$. Obviously, the $K$-transform is linear. It acts to the set of all measurable cylinder functions $F: \Gamma \rightarrow \mathbb{R}$, that is satisfying $F(\gamma)=F\left(\gamma_{\Lambda}\right)$ for some compact $\Lambda \subset \mathbb{R}^{d}$, where $\gamma_{\Lambda}=\gamma \cap \Lambda$. See [12] for more details. It is also invertible with the inverse given by

$$
\left(K^{-1} F\right)(\eta)=\sum_{\xi \subset \eta}(-1)^{|\eta \backslash \xi|} F(\xi) .
$$

For $G_{1}, G_{2} \in B_{b s}\left(\Gamma_{0}\right)$, it is known that

$$
\left(K G_{1}\right) \cdot\left(K G_{2}\right)=K\left(G_{1} \star G_{2}\right),
$$

where $G_{1} \star G_{2}$ is the "convolution" given by the formula

$$
\left(G_{1} \star G_{2}\right)(\eta)=\sum_{\xi \subset \eta} G_{1}(\xi) \sum_{\zeta \subset \xi} G_{2}(\eta \backslash \xi \cup \zeta) \in B_{b s}\left(\Gamma_{0}\right) .
$$

Denote

$$
e(f, \gamma)=\prod_{x \in \gamma} f(x)
$$

For a measurable compactly supported function $f: \mathbb{R}^{d} \rightarrow \mathbb{R}$, the following holds

$$
K(e(f, \cdot))(\gamma)=e(1+f, \gamma)
$$

A probability measure $\mu$ on $(\Gamma, \mathcal{B}(\Gamma))$ is said to have finite local moments of all orders if for any $n \in \mathbb{N}$ and a bounded Borel $\Lambda \subset \mathbb{R}^{d}$,

$$
\int_{\Gamma_{0}}\left|\gamma_{\Lambda}\right|^{n} \mu(d \gamma)<\infty
$$

where $|\eta|$ stands for the cardinality of $\eta \in \Gamma_{0}$. For such a measure $\mu$, one can define a correlation measure, $\rho_{\mu}$, on $\left(\Gamma_{0}, \mathcal{B}\left(\Gamma_{0}\right)\right)$ by

$$
\int_{\Gamma_{0}} G(\eta) \rho_{\mu}(d \eta)=\int_{\Gamma}(K G)(\gamma) \mu(d \gamma), \quad G \in B_{b s}\left(\Gamma_{0}\right) .
$$


By $\lambda$ we denote the Lebesgue-Poisson measure on $\left(\Gamma_{0}, \mathcal{B}\left(\Gamma_{0}\right)\right)$, that is, the correlation measure for the homogeneous Poisson measure with unit intensity. The Lebesgue-Poisson measure is uniquely defined by the following formula

$$
\int_{\Gamma_{0}} G(\eta) \lambda(d \eta)=G^{(0)}+\sum_{n=1}^{\infty} \frac{1}{n !} \int_{\left(\mathbb{R}^{d}\right)^{n}} G^{(n)}\left(x_{1}, x_{2}, \ldots, x_{n}\right) d x_{1} d x_{2} \cdots d x_{n}
$$

which has to hold for all $G \in B_{b s}\left(\Gamma_{0}\right)$. The Minlos lemma (see, e.g., [7, eq. $(2.2)])$, states that

$$
\begin{aligned}
& \int_{\Gamma_{0}} \ldots \int_{\Gamma_{0}} G\left(\eta_{1} \cup \eta_{2} \cup \cdots \cup \eta_{n}\right) H\left(\eta_{1}, \eta_{2}, \ldots, \eta_{n}\right) \lambda\left(d \eta_{1}\right) \lambda\left(d \eta_{2}\right) \ldots \lambda\left(d \eta_{n}\right) \\
& =\int_{\Gamma_{0}} G(\eta) \sum H\left(\eta_{1}, \eta_{2}, \ldots, \eta_{n}\right) \lambda(d \eta)
\end{aligned}
$$

where $n$ is a positive integer, $G: \Gamma_{0} \rightarrow \mathbb{R}, H:\left(\Gamma_{0}\right)^{n} \rightarrow \mathbb{R}$ are positive and measurable and the sum is taken over all $n$-part partitions $\left(\eta_{1}, \ldots, \eta_{n}\right)$ of $\eta$, where parts being empty configurations are also considered. For $n=2$, we can rewrite $(2.7)$ in the following form

$$
\int_{\Gamma_{0}} \int_{\Gamma_{0}} G(\eta \cup \xi) H(\eta, \xi) \lambda(d \eta) \lambda(d \xi)=\int_{\Gamma_{0}} G(\eta) \sum_{\xi \subset \eta} H(\xi, \eta \backslash \xi) \lambda(d \eta) .
$$

By taking

$$
H\left(\eta_{1}, \eta_{2}\right)= \begin{cases}h\left(x, \eta_{2}\right), & \eta_{1}=\{x\} \\ 0, & \left|\eta_{1}\right| \neq 1\end{cases}
$$

and using (2.6) we obtain the following special case of the Minlos lemma

$$
\iint_{\Gamma_{0}} G(\eta \cup x) h(x, \eta) d x \lambda(d \eta)=\int_{\mathbb{R}^{d}} \sum_{x \in \eta} G(\eta) h(x, \eta \backslash x) \lambda(d \eta) .
$$

Analogously, for

$$
H\left(\eta_{1}, \eta_{2}, \eta_{3}\right)= \begin{cases}h\left(x, y, \eta_{3}\right), & \eta_{1}=\{x\}, \eta_{2}=\{y\} \\ 0, & \left|\eta_{1}\right| \neq 1 \text { or }\left|\eta_{2}\right| \neq 1\end{cases}
$$

we have

$$
\begin{array}{r}
\frac{1}{2} \int_{\Gamma_{0}} \int_{\mathbb{R}^{d}} \int_{\mathbb{R}^{d}} G(\eta \cup\{x, y\}) h(x, y, \eta) d x d y \lambda(d \eta) \\
=\int_{\Gamma_{0}} \sum_{\{x, y\} \subset \eta} G(\eta) h(x, y, \eta \backslash\{x, y\}) \lambda(d \eta) .
\end{array}
$$


3. Dynamics of the correlation functions. In this section, we follow the approach described in [10], obtaining operator $L^{\Delta}$ acting on correlation functions, corresponding to (1.1). First, by using the $K$-transform (2.1) we obtain the operator $\hat{L}$ (see (3.4) below) and then $L^{\Delta}$ related to $\hat{L}$ by

$$
\int_{\Gamma_{0}}(\hat{L} G)(\eta) k(\eta) \lambda(d \eta)=\int_{\Gamma_{0}} G(\eta)\left(L^{\Delta} k\right)(\eta) \lambda(d \eta) .
$$

Recall, that

$$
L=L_{1}+L_{2}
$$

where

$$
L_{1} F(\gamma)=\sum_{\{x, y\} \subset \gamma} \int_{\mathbb{R}^{d}} \tilde{c}_{1}(x, y ; z ; \gamma)(F(\gamma \backslash\{x, y\} \cup z)-F(\gamma)) d z
$$

and

$$
L_{2} F(\gamma)=\sum_{x \in \gamma} \int_{\mathbb{R}^{d}} \tilde{c}_{2}(x ; y ; \gamma)(F(\gamma \backslash x \cup y)-F(\gamma)) d y .
$$

We assume that (see notion (2.4) for definition of $e(f, \gamma)$ )

$$
\begin{aligned}
\tilde{c}_{1}(x, y ; z ; \gamma) & =c_{1}(x, y ; z) e\left(t_{z}^{(1)}, \gamma \backslash\{x, y\}\right), \\
\tilde{c}_{2}(x ; y ; \gamma) & =c_{2}(x ; y) e\left(t_{y}^{(2)}, \gamma \backslash x\right),
\end{aligned}
$$

with

$$
t_{z}^{(1)}(u)=e^{-\phi_{1}(z-u)}, t_{y}^{(2)}(u)=e^{-\phi_{2}(y-u)} .
$$

and $c_{1}, c_{2}, \phi_{1}, \phi_{2}$ being positive real functions.

The above assumptions are important part of the model, as they guarantee that both jumping and coalescing are repulsive. Indeed, if $\gamma \backslash\{x, y\}$ is nonempty and at least one of its elements, say $u$, is such that $z-u$ lies in the support of $\phi_{1}$, then the coalescence rate $\tilde{c}_{1}(x, y ; z ; \gamma)$ is smaller than $c_{1}(x, y ; z)$. If there are no such elements in $\gamma \backslash\{x, y\}$, then $\tilde{c}_{1}(x, y ; z ; \gamma)=$ $c_{1}(x, y ; z)$. It means that the particles which are not involved in the coalescence, may influence it and this influence is repulsive. The analogous reasoning is valid for the jump rate $\tilde{c}_{2}(x ; y ; \gamma)$.

We add some technical assumptions listed below. Namely, let

$$
\begin{aligned}
\tilde{c}_{1}(x, y ; z ; \gamma) & =\left(K C_{x, y ; z}^{1}\right)(\gamma \backslash\{x, y\}), \\
\tilde{c}_{2}(x ; y ; \gamma) & =\left(K C_{x ; y}^{2}\right)(\gamma \backslash x)
\end{aligned}
$$


for some $C_{x, y ; z}^{1}$ and $C_{x ; y}^{2}$. We discuss the form of these functions later in this section. Additionally,

$$
\begin{aligned}
& c_{1}(x, y ; z)=c_{1}(y, x ; z), \\
& \int_{\left(\mathbb{R}^{d}\right)^{2}} c_{1}\left(x_{1}, x_{2} ; x_{3}\right) d x_{i} d x_{j}=\left\langle c_{1}\right\rangle<\infty, i, j=1,2,3, i \neq j, \\
& \int_{\mathbb{R}^{d}} c_{2}(x ; y) d x=\int_{\mathbb{R}^{d}} c_{2}(x ; y) d y=\left\langle c_{2}\right\rangle<\infty, \\
& \int_{\mathbb{R}^{d}} \phi_{1}(x) d x=\left\langle\phi_{1}\right\rangle<\infty, \quad \int_{\mathbb{R}^{d}} \phi_{2}(x) d x=\left\langle\phi_{2}\right\rangle<\infty .
\end{aligned}
$$

Suppose that $F=K G$, where $G: \Gamma_{0} \rightarrow \mathbb{R}$. Then, by writing $K \hat{L} G=L F$, we define

$$
\hat{L}=K^{-1} L K
$$

By the properties of the $K$-transform we derive an explicit formula for $\hat{L}$.

Proposition 3.1. $\hat{L}$ defined as above has the following form

$$
\begin{aligned}
\hat{L} G(\eta)= & \int_{\mathbb{R}^{d}} \sum_{\{x, y\} \subset \eta}\left[C_{x, y ; z}^{1} \star H_{x, y ; z}^{1}\right](\eta \backslash\{x, y\}) d z \\
& +\int_{\mathbb{R}^{d}} \sum_{x \in \eta}\left[C_{x ; y}^{2} \star H_{x ; y}^{2}\right](\eta \backslash x) d y
\end{aligned}
$$

where

$$
\begin{aligned}
H_{x, y ; z}^{1}(\eta) & =G(\eta \cup z)-G(\eta \cup x)-G(\eta \cup y)-G(\eta \cup\{x, y\}), \\
H_{x ; y}^{2}(\eta) & =G(\eta \cup y)-G(\eta \cup x) .
\end{aligned}
$$

The next step is to pass with the action of the operator $\hat{L}$ to the correlation functions, i.e. to obtain $L^{\Delta}$. The latter is defined by the pairing $\langle\langle\hat{L} G, k\rangle\rangle=\left\langle\left\langle G, L^{\Delta} k\right\rangle\right\rangle$, see (3.1). Let us consider the integral on the left hand side of equation (3.1). Using the Minlos lemma (2.7), we can transform it so that we can obtain $L^{\Delta}$.

Proposition 3.2. $L^{\Delta}$ defined as above is of the form

$$
L^{\Delta}=L_{1}^{\Delta}+L_{2}^{\Delta},
$$


where

$$
\begin{aligned}
L_{1}^{\Delta} k(\eta)= & \frac{1}{2} \int_{\left(\mathbb{R}^{d}\right)^{2}} \int_{\Gamma_{0}} \sum_{z \in \eta} c_{1}(x, y ; z) k(\eta \backslash z \cup \xi \cup\{x, y\}) \\
& \times e\left(t_{z}^{(1)}-1, \xi\right) e\left(t_{z}^{(1)}, \eta \backslash z\right) \lambda(d \xi) d x d y \\
- & \frac{1}{2} \int_{\left(\mathbb{R}^{d}\right)^{2}} \int_{\Gamma_{0}} \sum_{x \in \eta} c_{1}(x, y ; z) k(\eta \cup \xi \cup y) \\
& \times e\left(t_{z}^{(1)}-1, \xi\right) e\left(t_{z}^{(1)}, \eta \backslash x\right) \lambda(d \xi) d y d z \\
- & \frac{1}{2} \int_{\left(\mathbb{R}^{d}\right)^{2}} \int_{\Gamma_{0}} \sum_{y \in \eta} c_{1}(x, y ; z) k(\eta \cup \xi \cup x) \\
& \times e\left(t_{z}^{(1)}-1, \xi\right) e\left(t_{z}^{(1)}, \eta \backslash y\right) \lambda(d \xi) d x d z \\
- & \int_{\mathbb{R}^{d}} \int_{\Gamma_{0}} \sum_{\{x, y\} \subset \eta} c_{1}(x, y ; z) k(\eta \cup \xi) \\
& \times e\left(t_{z}^{(1)}-1, \xi\right) e\left(t_{z}^{(1)}, \eta \backslash\{x, y\}\right) \lambda(d \xi) d z
\end{aligned}
$$

and

$$
\begin{aligned}
L_{2}^{\Delta} k(\eta)= & \int_{\mathbb{R}^{d}} \int_{\Gamma_{0}} \sum_{y \in \eta} k(\eta \backslash y \cup \xi \cup x) c_{2}(x ; y) \\
& \times e\left(t_{y}^{(2)}-1, \xi\right) e\left(t_{y}^{(2)}-1, \eta \backslash y\right) \lambda(d \xi) d x \\
& -\int_{\mathbb{R}^{d}} \int_{\Gamma_{0}} k(\eta \cup \xi) \sum_{x \in \eta} c_{2}(x ; y) \\
& \times \prod_{u \in \xi} e\left(t_{y}^{(2)}-1, \xi\right) e\left(t_{y}^{(2)}-1, \eta \backslash x\right) \lambda(d \xi) d y .
\end{aligned}
$$

Proof of Proposition 3.1. First let us rewrite the operator $L$ in a more convenient form. Using (3.3) and recalling that any configuration treated as a subset of $\mathbb{R}^{d}$ is Lebesgue measure-zero as it is countable, we have

$$
\begin{aligned}
L_{1} F(\gamma)= & \sum_{\{x, y\} \subset \gamma} \int_{\mathbb{R}^{d} \backslash \gamma}\left(K C_{x, y ; z}^{1}(\cdot)[K G(\cdot \cup z)\right. \\
& -K G(\cdot \cup\{x, y\})])(\gamma \backslash\{x, y\}) d z .
\end{aligned}
$$

Observe that for any $\xi \in \Gamma, x, y, z \notin \xi$ we have

$$
K G(\xi \cup z)=\sum_{\eta \Subset \xi \cup z} G(\eta)=\sum_{\eta \Subset \xi}[G(\eta)+G(\eta \cup z)]=K[G(\cdot)+G(\cdot \cup z)](\xi)
$$


and analogously

$$
K G(\xi \cup\{x, y\})=K[G(\cdot)+G(\cdot \cup x)+G(\cdot \cup y)+G(\cdot \cup\{x, y\})](\xi) .
$$

Using linearity of the $K$-transform and above observations, we obtain

$$
\begin{aligned}
L_{1} F(\gamma)= & \sum_{\{x, y\} \subset \gamma} \int_{\mathbb{R}^{d}}\left(K C_{x, y ; z}^{1}(\cdot) K[G(\cdot \cup z)-G(\cdot \cup x)\right. \\
& -G(\cdot \cup y)-G(\cdot \cup\{x, y\})](\cdot))(\gamma \backslash\{x, y\}) d z .
\end{aligned}
$$

Considering the second part of the operator, we have

$$
\begin{aligned}
L_{2} F(\gamma) & =\sum_{x \in \gamma} \int_{\mathbb{R}^{d}} K C_{x ; y}^{2}(\gamma \backslash x)[K G(\gamma \backslash x \cup y)-K G(\gamma)] d y \\
& =\sum_{x \in \gamma} \int_{\mathbb{R}^{d}}\left(K C_{x ; y}^{2}(\cdot) K[G(\cdot \cup y)-G(\cdot \cup x)](\cdot)\right)(\gamma \backslash x) d y
\end{aligned}
$$

Using notion (3.5) and property (2.2) of the product of $K$-transforms, we derive

$$
\begin{gathered}
L_{1} F(\gamma)=\sum_{\{x, y\} \subset \gamma} \int_{\mathbb{R}^{d}} K\left[C_{x, y ; z}^{1} \star H_{x, y ; z}^{1}\right](\gamma \backslash\{x, y\}) d z \\
L_{2} F(\gamma)=\sum_{x \in \gamma} \int_{\mathbb{R}^{d}} K\left[C_{x ; y}^{2} \star H_{x ; y}^{2}\right](\gamma \backslash x) d y
\end{gathered}
$$

Therefore

$$
\begin{aligned}
L F(\gamma)= & \sum_{\{x, y\} \subset \gamma} \int_{\mathbb{R}^{d}} K\left[C_{x, y ; z}^{1} \star H_{x, y ; z}^{1}\right](\gamma \backslash\{x, y\}) d z \\
& +\sum_{x \in \gamma} \int_{\mathbb{R}^{d}} K\left[C_{x ; y}^{2} \star H_{x ; y}^{2}\right](\gamma \backslash x) d y .
\end{aligned}
$$

Recalling the definition (3.4) of the operator $\hat{L}$ and denoting

$$
\hat{L}_{1} G(\eta)=K^{-1} L_{1} F(\eta), \quad \hat{L}_{2} G(\eta)=K^{-1} L_{2} F(\eta),
$$


we obtain

$$
\begin{aligned}
\hat{L}_{1} G(\eta) & =\sum_{\xi \subset \eta}(-1)^{|\eta \backslash \xi|} \sum_{\{x, y\} \subset \xi} \int_{\mathbb{R}^{d}} K\left[C_{x, y ; z}^{1} \star H_{x, y ; z}^{1}\right](\xi \backslash\{x, y\}) d z \\
& =\int_{\mathbb{R}^{d}} \sum_{\{x, y\} \subset \eta} \sum_{\xi \subset \eta \backslash\{x, y\}}(-1)^{|\eta \backslash\{x, y\} \backslash \xi|} K\left[C_{x, y ; z}^{1} \star H_{x, y ; z}^{1}\right](\xi) d z \\
& =\int_{\mathbb{R}^{d}} \sum_{\{x, y\} \subset \eta} K^{-1} K\left[C_{x, y ; z}^{1} \star H_{x, y ; z}^{1}\right](\eta \backslash\{x, y\}) d z \\
& =\int_{\mathbb{R}^{d}} \sum_{\{x, y\} \subset \eta}\left[C_{x, y ; z}^{1} \star H_{x, y ; z}^{1}\right](\eta \backslash\{x, y\}) d z
\end{aligned}
$$

and analogously

$$
\begin{aligned}
\hat{L}_{2} G(\eta) & =\sum_{\xi \subset \eta}(-1)^{|\eta \backslash \xi|} \sum_{x \in \xi} \int_{\mathbb{R}^{d}} K\left[C_{x ; y}^{2} \star H_{x ; y}^{2}\right](\xi \backslash x) d y \\
& =\int_{\mathbb{R}^{d}} \sum_{x \in \eta} \sum_{\xi \subset \eta \backslash x}(-1)^{|\eta \backslash x \backslash \xi|} K\left[C_{x ; y}^{2} \star H_{x ; y}^{2}\right](\xi) d y \\
& =\int_{\mathbb{R}^{d}} \sum_{x \in \eta} K^{-1} K\left[C_{x ; y}^{2} \star H_{x ; y}^{2}\right](\eta \backslash x) d y \\
& =\int_{\mathbb{R}^{d}} \sum_{x \in \eta}\left[C_{x ; y}^{2} \star H_{x ; y}^{2}\right](\eta \backslash x) d y .
\end{aligned}
$$

Therefore

$$
\begin{aligned}
\hat{L} G(\eta)= & \int_{\mathbb{R}^{d}} \sum_{\{x, y\} \subset \eta}\left[C_{x, y ; z}^{1} \star H_{x, y ; z}^{1}\right](\eta \backslash\{x, y\}) d z \\
& +\int_{\mathbb{R}^{d}} \sum_{x \in \eta}\left[C_{x ; y}^{2} \star H_{x ; y}^{2}\right](\eta \backslash x) d y .
\end{aligned}
$$

Proof of Proposition 3.2. Using the special case (2.10) of the Minlos lemma and proposition (3.1), we have

$$
\begin{aligned}
& \int_{\Gamma_{0}}\left(\hat{L}_{1} G\right)(\eta) k(\eta) \lambda(d \eta) \\
& =\int_{\Gamma_{0}} \int_{\mathbb{R}^{d}} \sum_{\{x, y\} \subset \eta}\left[C_{x, y ; z}^{1} \star H_{x, y ; z}^{1}\right](\eta \backslash\{x, y\}) k(\eta) d z \lambda(d \eta)
\end{aligned}
$$




$$
=\frac{1}{2} \int_{\left(\mathbb{R}^{d}\right)^{3}} \int_{\Gamma_{0}}\left[C_{x, y ; z}^{1} \star H_{x, y ; z}^{1}\right](\eta) k(\eta \cup\{x, y\}) \lambda(d \eta) d x d y d z
$$

Recalling the definition (2.3) of the convolution $\star$ and using the Minlos lemma in the form (2.8) twice, we obtain

$$
\begin{aligned}
\int_{\Gamma_{0}}\left(\hat{L}_{1} G\right)(\eta) k(\eta) \lambda(d \eta)= & \frac{1}{2} \int_{\left(\mathbb{R}^{d}\right)^{3}} \int_{\Gamma_{0}} \sum_{\xi \subset \eta} C_{x, y ; z}^{1}(\xi) \sum_{\zeta \subset \xi} H_{x, y ; z}^{1}(\eta \backslash \xi \cup \zeta) \\
& \times k(\eta \cup\{x, y\}) \lambda(d \eta) d x d y d z \\
= & \frac{1}{2} \int_{\left(\mathbb{R}^{d}\right)^{3}} \int_{\Gamma_{0}} \int_{\Gamma_{0}} C_{x, y ; z}^{1}(\xi) \sum_{\zeta \subset \xi} H_{x, y ; z}^{1}(\eta \cup \zeta) \\
& \times k(\eta \cup \xi \cup\{x, y\}) \lambda(d \eta) \lambda(d \xi) d x d y d z \\
= & \frac{1}{2} \int_{\left(\mathbb{R}^{d}\right)^{3}} \int_{\Gamma_{0}} \int_{\Gamma_{0}} C_{\Gamma_{0}} C_{x, y ; z}^{1}(\xi \cup \zeta) H_{x, y ; z}^{1}(\eta \cup \zeta) \\
& \times k(\eta \cup \xi \cup \zeta \cup\{x, y\}) \lambda(d \eta) \lambda(d \xi) \lambda(d \zeta) d x d y d z .
\end{aligned}
$$

Using again the Minlos lemma (2.8), but in the opposite direction, we have

$$
\begin{aligned}
\int_{\Gamma_{0}}\left(\hat{L}_{1} G\right)(\eta) k(\eta) \lambda(d \eta)= & \frac{1}{2} \int_{\left(\mathbb{R}^{d}\right)^{3}} \int_{\Gamma_{0}} \int_{\Gamma_{0}} \sum_{\zeta \subset \eta} C_{x, y ; z}^{1}(\xi \cup \zeta) H_{x, y ; z}^{1}(\eta) \\
& \times k(\eta \cup \xi \cup\{x, y\}) \lambda(d \eta) \lambda(d \xi) d x d y d z \\
= & \frac{1}{2} \int_{\left(\mathbb{R}^{d}\right)^{3}} \int_{\Gamma_{0}} H_{x, y ; z}^{1}(\eta)\left[\int_{\Gamma_{0}} k(\eta \cup \xi \cup\{x, y\})\right. \\
& \left.\times \sum_{\zeta \subset \eta} C_{x, y ; z}^{1}(\xi \cup \zeta) \lambda(d \xi)\right] \lambda(d \eta) d x d y d z .
\end{aligned}
$$

Let us rewrite above using the definition (3.5) of $H_{x, y ; z}^{1}(\eta)$.

$$
\begin{aligned}
\int_{\Gamma_{0}} & \left(\hat{L}_{1} G\right)(\eta) k(\eta) \lambda(d \eta) \\
= & \frac{1}{2} \int_{\left(\mathbb{R}^{d}\right)^{3}} \int_{\Gamma_{0}}[G(\eta \cup z)-G(\eta \cup x)-G(\eta \cup y)-G(\eta \cup\{x, y\})] \\
& \times\left[\int_{\Gamma_{0}} k(\eta \cup \xi \cup\{x, y\}) \sum_{\zeta \subset \eta} C_{x, y ; z}^{1}(\xi \cup \zeta) \lambda(d \xi)\right] \lambda(d \eta) d x d y d z .
\end{aligned}
$$


Using the special cases (2.9) and (2.10) of the Minlos lemma, we obtain

$$
\begin{aligned}
& \int_{\Gamma_{0}}\left(\hat{L}_{1} G\right)(\eta) k(\eta) \lambda(d \eta) \\
& =\int_{\Gamma_{0}} G(\eta)\left[\frac{1}{2} \int_{\left(\mathbb{R}^{d}\right)^{2}} \int_{\Gamma_{0}} \sum_{z \in \eta} k(\eta \backslash z \cup \xi \cup\{x, y\}) \sum_{\zeta \subset \eta \backslash z} C_{x, y ; z}^{1}(\xi \cup \zeta) \lambda(d \xi) d x d y\right. \\
& \quad-\frac{1}{2} \int_{\left(\mathbb{R}^{d}\right)^{2}} \int_{\Gamma_{0}} \sum_{x \in \eta} k(\eta \cup \xi \cup y) \sum_{\zeta \subset \eta \backslash x} C_{x, y ; z}^{1}(\xi \cup \zeta) \lambda(d \xi) d y d z \\
& \quad-\frac{1}{2} \int_{\left(\mathbb{R}^{d}\right)^{2}} \int_{\Gamma_{0}} \sum_{y \in \eta} k(\eta \cup \xi \cup x) \sum_{\zeta \subset \eta \backslash y} C_{x, y ; z}^{1}(\xi \cup \zeta) \lambda(d \xi) d x d z \\
& \left.\quad-\int_{\mathbb{R}^{d}} \int_{\Gamma_{0}} \sum_{\{x, y\} \subset \eta} k(\eta \cup \xi) \sum_{\zeta \subset \eta \backslash\{x, y\}} C_{x, y ; z}^{1}(\xi \cup \zeta) \lambda(d \xi) d z\right] \lambda(d \eta) .
\end{aligned}
$$

Employing the same technique to the second part of the operator $\hat{L}$, we derive

$$
\begin{aligned}
& \int_{\Gamma_{0}}\left(\hat{L}_{2} G\right)(\eta) k(\eta) \lambda(d \eta) \\
& =\int_{\left(\mathbb{R}^{d}\right)^{2}} \int_{\Gamma_{0}} \int_{\Gamma_{0}} \sum_{\zeta \subset \eta} C_{x ; y}^{2}(\xi \cup \zeta) H_{x ; y}^{2}(\eta) k(\eta \cup \xi \cup x) \lambda(d \eta) \lambda(d \xi) d x d y \\
& =\int_{\Gamma_{0}} G(\eta)\left[\iint_{\mathbb{R}^{d}} \sum_{\Gamma_{0}} k(\eta \backslash y \cup \xi \cup x) \sum_{\zeta \subset \eta \backslash y} C_{x ; y}^{2}(\xi \cup \zeta) \lambda(d \xi) d x\right. \\
& \left.-\int_{\mathbb{R}^{d}} \int_{\Gamma_{0}} k(\eta \cup \xi) \sum_{x \in \eta} \sum_{\zeta \subset \eta \backslash x} C_{x ; y}^{2}(\xi \cup \zeta) \lambda(d \xi) d y\right] \lambda(d \eta) .
\end{aligned}
$$

Therefore, we obtain

$$
L^{\Delta} k(\eta)=
$$

$$
\begin{aligned}
= & \frac{1}{2} \int_{\left(\mathbb{R}^{d}\right)^{2}} \int_{\Gamma_{0}} \sum_{z \in \eta} k(\eta \backslash z \cup \xi \cup\{x, y\}) \sum_{\zeta \subset \eta \backslash z} C_{x, y ; z}^{1}(\xi \cup \zeta) \lambda(d \xi) d x d y \\
& -\frac{1}{2} \int_{\left(\mathbb{R}^{d}\right)^{2}} \int_{\Gamma_{0}} \sum_{x \in \eta} k(\eta \cup \xi \cup y) \sum_{\zeta \subset \eta \backslash x} C_{x, y ; z}^{1}(\xi \cup \zeta) \lambda(d \xi) d y d z \\
& -\frac{1}{2} \int_{\left(\mathbb{R}^{d}\right)^{2}} \int_{\Gamma_{0}} \sum_{y \in \eta} k(\eta \cup \xi \cup x) \sum_{\zeta \subset \eta \backslash y} C_{x, y ; z}^{1}(\xi \cup \zeta) \lambda(d \xi) d x d z
\end{aligned}
$$




$$
\begin{aligned}
& -\int_{\mathbb{R}^{d}} \int_{\Gamma_{0}} \sum_{\{x, y\} \subset \eta} k(\eta \cup \xi) \sum_{\zeta \subset \eta \backslash\{x, y\}} C_{x, y ; z}^{1}(\xi \cup \zeta) \lambda(d \xi) d z \\
& +\int_{\mathbb{R}^{d}} \int_{\Gamma_{0}} \sum_{y \in \eta} k(\eta \backslash y \cup \xi \cup x) \sum_{\zeta \subset \eta \backslash y} C_{x ; y}^{2}(\xi \cup \zeta) \lambda(d \xi) d x \\
& -\int_{\mathbb{R}^{d}} \int_{\Gamma_{0}} \sum_{x \in \eta} k(\eta \cup \xi) \sum_{\zeta \subset \eta \backslash x} C_{x ; y}^{2}(\xi \cup \zeta) \lambda(d \xi) d y .
\end{aligned}
$$

Note that so far we have not used any assumption about coefficients $\tilde{c}_{1}$ and $\tilde{c}_{2}$ apart from that they can be written as results of action of the $K$-transform on corresponding functions $C_{x, y ; z}^{1}$ and $C_{x ; y}^{2}$. Let us calculate explicit forms of these functions. Recall that

$$
\begin{gathered}
\tilde{c}_{1}(x, y ; z ; \gamma)=c_{1}(x, y ; z) \prod_{u \in \gamma \backslash\{x, y\}} e^{-\phi_{1}(z-u)}, \\
\tilde{c}_{2}(x ; y ; \gamma)=c_{2}(x ; y) \prod_{u \in \gamma \backslash x} e^{-\phi_{2}(y-u)} .
\end{gathered}
$$

We have

$$
K C_{x, y ; z}^{1}=c_{1}(x, y ; z) e\left(t_{z}^{(1)}, \cdot\right)
$$

that is

$$
\begin{aligned}
C_{x, y ; z}^{1} & =K^{-1} c_{1}(x, y ; z) e\left(1+t_{z}^{(1)}-1, \cdot\right)=c_{1}(x, y ; z) K^{-1} \sum_{\xi \subset \cdot} e\left(t_{z}^{(1)}-1, \xi\right) \\
& =c_{1}(x, y ; z) K^{-1} K e\left(t_{z}^{(1)}-1, \cdot\right)=c_{1}(x, y ; z) e\left(t_{z}^{(1)}-1, \cdot\right) .
\end{aligned}
$$

Therefore

$$
C_{x, y ; z}^{1}(\eta)=c_{1}(x, y ; z) e\left(t_{z}^{(1)}-1, \eta\right)
$$

Analogously we can derive

$$
C_{x ; y}^{2}(\eta)=c_{2}(x ; y) e\left(t_{y}^{(2)}-1, \eta\right)
$$

Using the above, we can rewrite the operator $L^{\Delta}$. For convenience let us denote the part of it corresponding to the coalescence, that is the terms (3.6)-(3.9), as $L_{1}^{\Delta}$ and the part corresponding to the jumps, that is the 
terms (3.10)-(3.11), as $L_{2}^{\Delta}$. Substituting (3.12), we derive

$$
\begin{aligned}
& L_{1}^{\Delta} k(\eta)=\frac{1}{2} \int_{\left(\mathbb{R}^{d}\right)^{2}} \int_{\Gamma_{0}} \sum_{z \in \eta} k(\eta \backslash z \cup \xi \cup\{x, y\}) \sum_{\zeta \subset \eta \backslash z} c_{1}(x, y ; z) \\
& \quad \times e\left(t_{z}^{(1)}-1, \xi \cup \zeta\right) \lambda(d \xi) d x d y \\
& -\frac{1}{2} \int_{\left(\mathbb{R}^{d}\right)^{2}} \int_{\Gamma_{0}} \sum_{x \in \eta} k(\eta \cup \xi \cup y) \sum_{\zeta \subset \eta \backslash x} c_{1}(x, y ; z) e\left(t_{z}^{(1)}-1, \xi \cup \zeta\right) \lambda(d \xi) d y d z \\
& -\frac{1}{2} \int_{\left(\mathbb{R}^{d}\right)^{2}} \int_{\Gamma_{0}} \sum_{y \in \eta} k(\eta \cup \xi \cup x) \sum_{\zeta \subset \eta \backslash y} c_{1}(x, y ; z) e\left(t_{z}^{(1)}-1, \xi \cup \zeta\right) \lambda(d \xi) d x d z \\
& -\int_{\mathbb{R}^{d}} \int_{\Gamma_{0}} \sum_{\{x, y\} \subset \eta} k(\eta \cup \xi) \sum_{\zeta \subset \eta \backslash\{x, y\}} c_{1}(x, y ; z) e\left(t_{z}^{(1)}-1, \xi \cup \zeta\right) \lambda(d \xi) d z
\end{aligned}
$$

and analogously using (3.13), we obtain

$$
\begin{aligned}
L_{2}^{\Delta} k(\eta)= & \int_{\mathbb{R}^{d}} \int_{\Gamma_{0}} \sum_{y \in \eta} k(\eta \backslash y \cup \xi \cup x) \sum_{\zeta \subset \eta \backslash y} c_{2}(x ; y) e\left(t_{y}^{(2)}-1, \xi \cup \zeta\right) \lambda(d \xi) d x \\
& -\int_{\mathbb{R}^{d}} \int_{\Gamma_{0}} k(\eta \cup \xi) \sum_{x \in \eta} \sum_{\zeta \subset \eta \backslash x} c_{2}(x ; y) e\left(t_{y}^{(2)}-1, \xi \cup \zeta\right) \lambda(d \xi) d y .
\end{aligned}
$$

Consider the first component of $L_{1}^{\Delta}$ and denote it as

$$
\begin{aligned}
L_{11}^{\Delta} k(\eta)= & \frac{1}{2} \int_{\left(\mathbb{R}^{d}\right)^{2}} \int_{\Gamma_{0}} \sum_{z \in \eta} k(\eta \backslash z \cup \xi \cup\{x, y\}) \\
& \times \sum_{\zeta \subset \eta \backslash z} c_{1}(x, y ; z) e\left(t_{z}^{(1)}-1, \xi \cup \zeta\right) \lambda(d \xi) d x d y .
\end{aligned}
$$

Next, for a given $\eta$ let us introduce $C(\eta)=\left\{\xi \in \Gamma_{0}: \xi \cap \eta \neq \emptyset\right\}$. Then, because any configuration treated as a measurable subset of $\mathbb{R}^{d}$ is of Lebesgue measure 0 and the empty configuration does not belong to $C(\eta)$ for any $\eta \in \Gamma_{0}$, we have $\lambda(C(\eta))=0$ for every $\eta \in \Gamma_{0}$. Indeed, using the characterization (2.6) of the integral w.r.t. the Lebesgue-Poisson measure, we obtain

$$
\lambda(C(\eta))=\int_{\Gamma_{0}} I_{C(\eta)}(\xi) \lambda(d \xi)=I_{C(\eta)}^{(0)}+\sum_{n=1}^{\infty} \frac{1}{n !} \int_{\left(\mathbb{R}^{d}\right)^{n}} I_{C(\eta)}^{(n)}\left(x_{1}, \ldots, x_{n}\right) d x_{1} \ldots d x_{n} .
$$

First, notice that $I_{C(\eta)}^{(0)}=0$, as empty configuration cannot have common part with any configuration. Then, because

$$
I_{C(\eta)}^{(n)}\left(x_{1}, \ldots, x_{n}\right) \leq I_{C(\eta)}^{(1)}\left(x_{1}\right)+I_{C(\eta)}^{(1)}\left(x_{2}\right)+\cdots+I_{C(\eta)}^{(1)}\left(x_{n}\right)
$$


we have for every $n \in \mathbb{N}$

$$
\int_{\left(\mathbb{R}^{d}\right)^{n}} I_{C(\eta)}^{(n)}\left(x_{1}, \ldots, x_{n}\right) d x_{1} \ldots d x_{n} \leq n \int_{\left(\mathbb{R}^{d}\right)^{n-1}}\left[\int_{\mathbb{R}^{d}} I_{C(\eta)}^{(1)}(x) d x\right] d x_{1} \ldots d x_{n-1} .
$$

Taking into account that

$$
\int_{\mathbb{R}^{d}} I_{C(\eta)}^{(1)}(x) d x=\int_{\mathbb{R}^{d}} I_{\eta}(x) d x=l(\eta)=0,
$$

where $l$ denotes the Lebesgue measure, one can clearly see that $\lambda(C(\eta))=0$. Therefore, when integrating over $\Gamma_{0} \backslash C(\eta)$ instead of $\Gamma_{0}$, the result is the same. However, all subconfigurations $\zeta$ of $\eta$ are disjoint with any $\xi \in$ $\Gamma_{0} \backslash C(\eta)$, which allows us to separate the product taken over $\xi \cup \zeta$ into one taken over $\xi$ and another taken over $\zeta$. Thus we can write

$$
\begin{aligned}
L_{11}^{\Delta} k(\eta)= & \frac{1}{2} \int_{\left(\mathbb{R}^{d}\right)^{2}} \int_{\Gamma_{0}} \sum_{z \in \eta} c_{1}(x, y ; z) k(\eta \backslash z \cup \xi \cup\{x, y\}) \\
& \times e\left(t_{z}^{(1)}-1, \xi\right) \sum_{\zeta \subset \eta \backslash z} e\left(t_{z}^{(1)}-1, \zeta\right) \lambda(d \xi) d x d y .
\end{aligned}
$$

Recalling the definition (2.1) of the $K$-transform and its property (2.5), we have

$$
\sum_{\zeta \subset \eta \backslash z} e\left(t_{z}^{(1)}-1, \zeta\right)=K\left(e\left(t_{z}^{(1)}-1, \cdot\right)\right)(\eta \backslash z)=e\left(t_{z}^{(1)}, \eta \backslash z\right) .
$$

Therefore we can rewrite the action of $L_{11}^{\Delta}$ in the form

$$
\begin{aligned}
L_{11}^{\Delta} k(\eta)= & \frac{1}{2} \int_{\left(\mathbb{R}^{d}\right)^{2}} \int_{\Gamma_{0}} \sum_{z \in \eta} c_{1}(x, y ; z) k(\eta \backslash z \cup \xi \cup\{x, y\}) \\
& \times e\left(t_{z}^{(1)}-1, \xi\right) e\left(t_{z}^{(1)}, \eta \backslash z\right) \lambda(d \xi) d x d y .
\end{aligned}
$$

Applying the same method for the rest of the $L_{1}^{\Delta}$ and for the $L_{2}^{\Delta}$, we obtain the result.

\section{The Vlasov scaling and the kinetic equation.}

4.1. The Vlasov scaling. We follow the scaling technique described in [8]. Let us introduce the scale parameter $\epsilon \in[0,1]$ with $\epsilon=1$ corresponding to the unscaled case (microscopic level) and $\epsilon \rightarrow 0$ to the fully rescaled case, where the description of the system is based on the density of a medium (mesoscopic level), instead of the corpuscular structure. Evolution of density in time is characterized by the corresponding kinetic equation. The scaling is performed in such a way that the evolution of the rescaled case preserves poissonity of a correlation function. This allows us to identify above abstract density of the medium with density of the Poisson measure, which is concrete 
mathematical object. We alter the operator $L^{\Delta}$ by scaling $c_{1} \rightarrow \epsilon c_{1}, \phi_{1} \rightarrow$ $\epsilon \phi_{1}$ and $\phi_{2} \rightarrow \epsilon \phi_{2}$ for $\epsilon \in(0,1]$, which can be interpreted as weakening the interactions between particles. Altered in such a way operator we denote by $L_{\epsilon}^{\Delta}$. Next, we renormalize it, defining

$$
L_{\epsilon}^{r e n} k(\eta)=\epsilon^{|\eta|} L_{\epsilon}^{\Delta}\left(\epsilon^{-|\eta|} k(\eta)\right) .
$$

Let us consider the first component of the operator $L_{\epsilon}^{\text {ren }}$. We have (cf. Proposition 3.2)

$$
\begin{aligned}
L_{11, \epsilon}^{r e n} k(\eta)= & \frac{1}{2} \epsilon^{|\eta|} \int_{\left(\mathbb{R}^{d}\right)^{2}} \int_{\Gamma_{0}} \sum_{z \in \eta} \epsilon c_{1}(x, y ; z) \epsilon^{-|\eta \backslash z \cup \xi \cup\{x, y\}|} k(\eta \backslash z \cup \xi \cup\{x, y\}) \\
& \times \prod_{u \in \xi}\left(e^{-\epsilon \phi_{1}(z-u)}-1\right) \prod_{u \in \eta \backslash z} e^{-\epsilon \phi_{1}(z-u)} \lambda(d \xi) d x d y .
\end{aligned}
$$

Note that integrating over $\left(\mathbb{R}^{d}\right)^{2} \backslash(\eta \times \eta)$ instead of $\left(\mathbb{R}^{d}\right)^{2}$ and over $\Gamma_{0} \backslash(\eta \cup$ $\{x, y\})$ instead of $\Gamma_{0}$ does not influence the result, so we have

$$
\begin{aligned}
L_{11, \epsilon}^{r e n} k(\eta)= & \frac{1}{2} \int_{\left(\mathbb{R}^{d}\right)^{2}} \int_{\Gamma_{0}} \sum_{z \in \eta} c_{1}(x, y ; z) k(\eta \backslash z \cup \xi \cup\{x, y\}) \\
& \times \prod_{u \in \xi} \frac{1}{\epsilon}\left(e^{-\epsilon \phi_{1}(z-u)}-1\right) \prod_{u \in \eta \backslash z} e^{-\epsilon \phi_{1}(z-u)} \lambda(d \xi) d x d y .
\end{aligned}
$$

Let us pass with $\epsilon$ to the limit. Noting that

$$
\lim _{\epsilon \rightarrow 0} \frac{1}{\epsilon}\left(e^{-\epsilon \phi_{1}(z-u)}-1\right)=-\phi_{1}(z-u),
$$

we can write

$$
\begin{aligned}
\lim _{\epsilon \rightarrow 0} L_{11, \epsilon}^{r e n} k(\eta)= & \frac{1}{2} \int_{\left(\mathbb{R}^{d}\right)^{2}} \int_{\Gamma_{0}} \sum_{z \in \eta} c_{1}(x, y ; z) k(\eta \backslash z \cup \xi \cup\{x, y\}) \\
& \times \prod_{u \in \xi}\left(-\phi_{1}(z-u)\right) \lambda(d \xi) d x d y .
\end{aligned}
$$

Let us denote $V=\lim _{\epsilon \rightarrow 0} L_{\epsilon}^{r e n}$. Calculating analogously as above, one derives

$$
\begin{aligned}
V k(\eta)= & \frac{1}{2} \int_{\left(\mathbb{R}^{d}\right)^{2}} \int_{\Gamma_{0}} \sum_{z \in \eta} c_{1}(x, y ; z) k(\eta \backslash z \cup \xi \cup\{x, y\}) \\
& \times \prod_{u \in \xi}\left(-\phi_{1}(z-u)\right) \lambda(d \xi) d x d y \\
& -\frac{1}{2} \int_{\left(\mathbb{R}^{d}\right)^{2}} \int_{\Gamma_{0}} \sum_{x \in \eta} c_{1}(x, y ; z) k(\eta \cup \xi \cup y) \prod_{u \in \xi}\left(-\phi_{1}(z-u)\right) \lambda(d \xi) d y d z
\end{aligned}
$$




$$
\begin{aligned}
& -\frac{1}{2} \int_{\left(\mathbb{R}^{d}\right)^{2}} \int_{\Gamma_{0}} \sum_{y \in \eta} c_{1}(x, y ; z) k(\eta \cup \xi \cup x) \prod_{u \in \xi}\left(-\phi_{1}(z-u)\right) \lambda(d \xi) d x d z \\
& +\int_{\mathbb{R}^{d}} \int_{\Gamma_{0}} \sum_{y \in \eta} k(\eta \backslash y \cup \xi \cup x) c_{2}(x ; y) \prod_{u \in \xi}\left(-\phi_{2}(y-u)\right) \lambda(d \xi) d x \\
& -\int_{\mathbb{R}^{d}} \int_{\Gamma_{0}} k(\eta \cup \xi) \sum_{x \in \eta} c_{2}(x ; y) \prod_{u \in \xi}\left(-\phi_{2}(y-u)\right) \lambda(d \xi) d y .
\end{aligned}
$$

Consider the following problem

$$
\frac{d}{d t} r_{t}=V r_{t}, \quad r_{t=0}=r_{0}
$$

in the Banach space

$$
\mathcal{K}_{\theta}=\left\{r: \Gamma_{0} \rightarrow \mathbb{R}:\|r\|_{\theta}<\infty\right\},
$$

where

$$
\|r\|_{\theta}=\underset{\eta \in \Gamma_{0}}{\operatorname{ess} \sup } e^{\theta|\eta|}|r(\eta)| .
$$

As usual in the Vlasov scaling, we assume that the initial state is a Poisson measure and hence $r_{0}$ is of the form

$$
r_{0}(\eta)=\prod_{x \in \eta} \rho_{0}(x) .
$$

If $r_{t}$ is of the product form

$$
r_{t}(\eta)=\prod_{x \in \eta} \rho_{t}(x)
$$

then

$$
\frac{d}{d t} r_{t}(\eta)=\frac{d}{d t} \prod_{x \in \eta} \rho_{t}(x)=\sum_{x \in \eta}\left(\prod_{y \in \eta \backslash x} \rho_{t}(y)\right) \frac{d}{d t} \rho_{t}(x) .
$$

Therefore, by expressing $V r_{t}$ in the form

$$
V r_{t}(\eta)=\sum_{x \in \eta}\left(\prod_{y \in \eta \backslash x} \rho_{t}(y)\right) v\left(\rho_{t}, x\right),
$$

for some $v\left(\rho_{t}, x\right)$ (which is done below), we can obtain a problem for $\rho_{t}$ corresponding to (4.1), namely a kinetic equation

$$
\frac{d}{d t} \rho_{t}(x)=v\left(\rho_{t}, x\right), \quad \rho_{t=0}=\rho_{0} .
$$

If $\rho_{t}$ is a solution of (4.2), then we can easily check that

$$
r_{t}(\eta)=\prod_{x \in \eta} \rho_{t}(x)
$$

is a solution of (4.1). Therefore by showing existence of solution of (4.2), we obtain the existence of (4.1). As we show the local existence and uniqueness 
of the kinetic equation (see Theorem 4.1 below), we obtain the local existence of (4.1). It is possible to show its uniqueness using the Ovsyannikov's method, but it is beyond the scope of this paper.

Let us denote the first component of $V$ by $V_{1}$. We have

$$
\begin{aligned}
V_{1} r_{t}(\eta)= & \frac{1}{2} \int_{\left(\mathbb{R}^{d}\right)^{2}} \int_{\Gamma_{0}} \sum_{z \in \eta} c_{1}(x, y ; z) r_{t}(\eta \backslash z \cup \xi \cup\{x, y\}) \\
& \times \prod_{u \in \xi}\left(-\phi_{1}(z-u)\right) \lambda(d \xi) d x d y \\
= & \frac{1}{2} \int_{\left(\mathbb{R}^{d}\right)^{2}} \int_{\Gamma_{0}} \sum_{z \in \eta} c_{1}(x, y ; z) \prod_{v \in \eta \backslash z \cup \xi \cup\{x, y\}} \rho_{t}(v) \\
& \times \prod_{u \in \xi}\left(-\phi_{1}(z-u)\right) \lambda(d \xi) d x d y .
\end{aligned}
$$

Because $l(\eta)=0$ and $\lambda(C(\eta \cup\{x, y\}))=0$, one can rewrite above as

$$
\begin{aligned}
V_{1} r_{t}(\eta)= & \sum_{z \in \eta}\left(\prod_{v \in \eta \backslash z} \rho_{t}(v)\right)\left(\frac{1}{2} \int_{\left(\mathbb{R}^{d}\right)^{2} \backslash(\eta \times \eta)} \int_{\Gamma_{0} \backslash C(\eta \cup\{x, y\})} c_{1}(x, y ; z)\right. \\
& \left.\times \rho_{t}(x) \rho_{t}(y) \prod_{u \in \xi}\left(-\rho_{t}(u) \phi_{1}(z-u)\right) \lambda(d \xi) d x d y\right) .
\end{aligned}
$$

Therefore

$$
V_{1} r_{t}(\eta)=\sum_{z \in \eta}\left(\prod_{v \in \eta \backslash z} \rho_{t}(v)\right) v_{1}\left(\rho_{t}, z\right)
$$

where

$$
v_{1}\left(\rho_{t}, z\right)=\frac{1}{2} \int_{\left(\mathbb{R}^{d}\right)^{2}} c_{1}(x, y ; z) \rho_{t}(x) \rho_{t}(y) \int_{\Gamma_{0}} \prod_{u \in \xi}\left(-\rho_{t}(u) \phi_{1}(z-u)\right) \lambda(d \xi) d x d y .
$$

Noting that for $a(u)=-\rho_{t}(u) \phi_{1}(z-u)$

$$
\begin{aligned}
\int_{\Gamma_{0}} \prod_{u \in \xi} a(u) \lambda(d \xi) & =1+\sum_{n=1}^{\infty} \frac{1}{n !} \int_{\left(\mathbb{R}^{d}\right)^{n}} a\left(x_{1}\right) \ldots a\left(x_{n}\right) d x_{1} \ldots d x_{n} \\
& =1+\sum_{n=1}^{\infty} \frac{1}{n !}\left(\int_{\mathbb{R}^{d}} a(u) d u\right)^{n}=\exp \left(\int_{\mathbb{R}^{d}} a(u) d u\right),
\end{aligned}
$$

we can reformulate the above, obtaining

$$
v_{1}\left(\rho_{t}, x\right)=\frac{1}{2} \int_{\left(\mathbb{R}^{d}\right)^{2}} c_{1}(y, z ; x) \rho_{t}(y) \rho_{t}(z) \exp \left(-\int_{\mathbb{R}^{d}} \phi_{1}(x-u) \rho_{t}(u) d u\right) d y d z .
$$


Calculating analogously as above, one can obtain explicit form of $v$ and thus the following kinetic equation

$$
\begin{aligned}
\frac{d}{d t} \rho_{t}(x)= & \frac{1}{2} \int_{\left(\mathbb{R}^{d}\right)^{2}} c_{1}(y, z ; x) \exp \left(-\int_{\mathbb{R}^{d}} \phi_{1}(x-u) \rho_{t}(u) d u\right) \rho_{t}(y) \rho_{t}(z) d y d z \\
& -\frac{1}{2} \int_{\left(\mathbb{R}^{d}\right)^{2}}\left(c_{1}(x, y ; z)+c_{1}(y, x ; z)\right) \\
& \quad \times \exp \left(-\int_{\mathbb{R}^{d}} \phi_{1}(z-u) \rho_{t}(u) d u\right) \rho_{t}(x) \rho_{t}(y) d y d z \\
& +\int_{\mathbb{R}^{d}} c_{2}(y ; x) \exp \left(-\int_{\mathbb{R}^{d}} \phi_{2}(x-u) \rho_{t}(u) d u\right) \rho_{t}(y) d y \\
& -\int_{\mathbb{R}^{d}} c_{2}(x ; y) \exp \left(-\int_{\mathbb{R}^{d}} \phi_{2}(y-u) \rho_{t}(u) d u\right) \rho_{t}(x) d y, \\
\rho_{t=0}= & \rho_{0} .
\end{aligned}
$$

4.2. The kinetic equation. Let us rewrite the problem (4.3) as

$$
\frac{d}{d t} \rho_{t}(x)=R_{1}\left(\rho_{t}, x\right)+R_{2}\left(\rho_{t}, x\right), \quad \rho_{t=0}(x)=\rho_{0}(x),
$$

where

$$
\begin{aligned}
R_{1}\left(\rho_{t}, x\right)= & -\frac{1}{2} \rho_{t}(x) \int_{\left(\mathbb{R}^{d}\right)^{2}}\left(c_{1}(x, y ; z)+c_{1}(y, x ; z)\right) \rho_{t}(y) d y d z \\
& -h\left(\rho_{t}, x\right) \int_{\mathbb{R}^{d}} c_{2}(x ; y) d y \\
= & -\rho_{t}(x) h\left(\rho_{t}, x\right)
\end{aligned}
$$

for

$$
h\left(\rho_{t}, x\right)=\frac{1}{2} \int_{\left(\mathbb{R}^{d}\right)^{2}}\left(c_{1}(x, y ; z)+c_{1}(y, x ; z)\right) \rho_{t}(y) d y d z+\left\langle c_{2}\right\rangle
$$

and

$$
\begin{aligned}
R_{2}\left(\rho_{t}, x\right)= & \frac{1}{2} \int_{\left(\mathbb{R}^{d}\right)^{2}} c_{1}(y, z ; x) \exp \left(-\int_{\mathbb{R}^{d}} \phi_{1}(x-u) \rho_{t}(u) d u\right) \rho_{t}(y) \rho_{t}(z) d y d z \\
& +\frac{1}{2} \int_{\left(\mathbb{R}^{d}\right)^{2}}\left(c_{1}(x, y ; z)+c_{1}(y, x ; z)\right)
\end{aligned}
$$




$$
\begin{aligned}
& \times\left[1-\exp \left(-\int_{\mathbb{R}^{d}} \phi_{1}(z-u) \rho_{t}(u) d u\right)\right] \rho_{t}(x) \rho_{t}(y) d y d z \\
+ & \int_{\mathbb{R}^{d}} c_{2}(y ; x) \exp \left(-\int_{\mathbb{R}^{d}} \phi_{2}(x-u) \rho_{t}(u) d u\right) \rho_{t}(y) d y \\
+ & \int_{\mathbb{R}^{d}} c_{2}(x ; y)\left[1-\exp \left(-\int_{\mathbb{R}^{d}} \phi_{2}(y-u) \rho_{t}(u) d u\right)\right] \rho_{t}(x) d y .
\end{aligned}
$$

Note that from (4.4) we can obtain the equivalent integral equation

$$
\begin{aligned}
\rho_{t}(x)= & \rho_{0}(x) \exp \left(-\int_{0}^{t} h\left(\rho_{s}, x\right) d s\right) \\
& +\int_{0}^{t} R_{2}\left(\rho_{s}, x\right) \exp \left(-\int_{s}^{t} h\left(\rho_{\sigma}, x\right) d \sigma\right) d s .
\end{aligned}
$$

Theorem 4.1. Problem (4.4) with the initial condition $\rho_{0} \in L^{\infty}\left(\mathbb{R}^{d}\right), \rho_{0} \geq$ 0 has the unique local classical solution.

Consider $X_{T}=C\left([0, T] \rightarrow L^{\infty}\left(\mathbb{R}^{d}\right)\right), T>0$ with the norm

$$
\|\rho\|_{T, \gamma}=\sup _{t \in[0, T]} e^{-\gamma\left\langle c_{2}\right\rangle t}\left\|\rho_{t}\right\|_{L^{\infty}}
$$

Denote

$$
\begin{aligned}
B_{T, \gamma}(r) & =\left\{\rho \in X_{T}:\|\rho\|_{T, \gamma} \leq r, \rho_{t} \geq 0 \forall t \in[0, T]\right\}, \\
B_{T, \gamma}\left(r, \rho_{0}\right) & =\left\{\psi \in B_{T, \gamma}(r): \psi_{0}=\rho_{0}\right\},
\end{aligned}
$$

where $\rho_{0} \in L^{\infty}\left(\mathbb{R}^{d}\right), \rho_{0} \geq 0, r \geq\left\|\rho_{0}\right\|_{L^{\infty}}$ and $T, \gamma>0$.

Lemma 4.2. Given $r>0$, there exist $\gamma, \tilde{T}>0$ such that $F$ defined by the RHS of (4.5) with the domain $B_{T^{*}, \gamma}(r) \subset X_{T^{*}}$ acts again to the $B_{T^{*}, \gamma}(r)$ for any $T^{*} \in[0, \tilde{T}]$.

Lemma 4.3. Let $\rho_{0} \in L^{\infty}\left(\mathbb{R}^{d}\right), \rho_{0} \geq 0$ and $r \geq\left\|\rho_{0}\right\|_{L^{\infty}}$. Let $\tilde{T}, \gamma$ satisfy Lemma 4.2 for this $r$. We can choose $T^{*} \in[0, \tilde{T}]$ in such a way that for any $\rho, \psi$ in $B_{T^{*}, \gamma}\left(r, \rho_{0}\right)$ the inequality $\|F(\rho)-F(\psi)\|_{T^{*}, \gamma} \leq C\|\rho-\psi\|_{T^{*}, \gamma}$ holds for some constant $C<1$.

Proof of Theorem 4.1. Choose $r>\left\|\rho_{0}\right\|_{L^{\infty}}$ and take corresponding $\gamma, \tilde{T}$ from Lemma 4.2. Take $T^{*}$ as in Lemma 4.3. Define the sequence of Picard iterations $\left(\rho^{(n)}\right)_{n \in \mathbb{N}_{0}}$ in the following way

$$
\begin{aligned}
& \rho_{t}^{(0)}=\rho_{0} \forall t \in\left[0, T^{*}\right], \\
& \rho^{(n)}=F\left(\rho^{(n-1)}\right), n \in \mathbb{N} .
\end{aligned}
$$


Obviously, $\rho^{(0)} \in B_{T^{*}, \gamma}(r)$. Therefore, by Lemma 4.2, $\rho^{(n)} \in B_{T^{*}, \gamma}(r)$ for all $n \in \mathbb{N}_{0}$ and from Lemma 4.3 we obtain

$\left\|\rho^{(n+k)}-\rho^{(n)}\right\|_{T^{*}, \gamma} \leq\left\|\rho^{(1)}-\rho^{(0)}\right\|_{T^{*}, \gamma} \sum_{i=1}^{k} C^{n+i-1} \leq\left\|\rho^{(1)}-\rho^{(0)}\right\|_{T^{*}, \gamma} \frac{C^{n}}{1-C}$,

where $C<1$ is a positive constant. Therefore $\left(\rho^{(n)}\right)_{n \in \mathbb{N}_{0}}$ defined by (4.6) is a Cauchy sequence. As $B_{T^{*}, \gamma}(r)$ is a closed subset of a Banach space, there exists

$$
\lim _{n \rightarrow \infty} \rho^{(n)}=\rho \in B_{T^{*}, \gamma}(r) .
$$

Clearly $F(\rho)=\rho$ and therefore $\rho_{t}$ satisfies the integral equation (4.5) for $t \in\left[0, T^{*}\right]$. Thus it is a local classical solution of (4.4).

Now suppose there is another local classical solution of this equation, say $\psi$. Then $\psi_{0}=\rho_{0}$ and for $r, \gamma, T^{*}$ as above, there exists $T \leq T^{*}$ such that $\psi \in B_{T, \gamma}(r)$. However, from Lemma 4.3 we have

$$
\|\rho-\psi\|_{T, \gamma}=\|F(\rho)-F(\psi)\|_{T, \gamma} \leq C\|\rho-\psi\|_{T, \gamma}
$$

for $C<1$, which means that

$$
\|\rho-\psi\|_{T, \gamma}=0
$$

and thus $\rho$ is the unique local classical solution.

Proof of Lemma 4.2. Take arbitrary $T, \gamma>0$ and $\rho \in B_{T, \gamma}(r)$. Note that

$$
\begin{aligned}
& h\left(\rho_{t}, x\right) \geq\left\langle c_{2}\right\rangle, \\
& R_{2}\left(\rho_{t}, x\right) \leq \frac{3}{2}\left\|\rho_{t}\right\|_{L^{\infty}}^{2}\left\langle c_{1}\right\rangle+2\left\|\rho_{t}\right\|_{L^{\infty}}\left\langle c_{2}\right\rangle, \\
& \rho_{t}(x) \leq\left\|\rho_{t}\right\|_{L^{\infty}} \leq e^{\gamma\left\langle c_{2}\right\rangle t}\|\rho\|_{T, \gamma} .
\end{aligned}
$$

It is obvious that $F$ preserves positiveness of $\rho$. Furthermore, using above estimates and the definition of $B_{T, \gamma}(r)$, we derive

$$
\begin{aligned}
(F(\rho))_{t}(x)= & \rho_{0}(x) \exp \left(-\int_{0}^{t} h\left(\rho_{s}, x\right) d s\right) \\
& +\int_{0}^{t} R_{2}\left(\rho_{s}, x\right) \exp \left(-\int_{s}^{t} h\left(\rho_{\sigma}, x\right) d \sigma\right) d s \\
\leq & \left\|\rho_{0}\right\|_{L^{\infty}} e^{-t\left\langle c_{2}\right\rangle}+\int_{0}^{t} R_{2}\left(\rho_{s}, x\right) e^{(s-t)\left\langle c_{2}\right\rangle} d s
\end{aligned}
$$




$$
\begin{aligned}
\leq & e^{-t\left\langle c_{2}\right\rangle}\left[\|\rho\|_{T, \gamma}+\int_{0}^{t}\left(\frac{3}{2}\left\langle c_{1}\right\rangle e^{(2 \gamma+1)\left\langle c_{2}\right\rangle s}\|\rho\|_{T, \gamma}^{2}\right.\right. \\
& \left.\left.+2\left\langle c_{2}\right\rangle e^{(\gamma+1)\left\langle c_{2}\right\rangle s}\|\rho\|_{T, \gamma}\right) d s\right] .
\end{aligned}
$$

Therefore, we obtain

$$
\begin{aligned}
\left\|(F(\rho))_{t}\right\|_{L^{\infty}} \leq & e^{-t\left\langle c_{2}\right\rangle} r\left[1+\frac{3\left\langle c_{1}\right\rangle r}{2(2 \gamma+1)\left\langle c_{2}\right\rangle}\left(e^{(2 \gamma+1)\left\langle c_{2}\right\rangle t}-1\right)\right. \\
& \left.+\frac{2}{\gamma+1}\left(e^{(\gamma+1)\left\langle c_{2}\right\rangle t}-1\right)\right] .
\end{aligned}
$$

Thus

$$
\|(F(\rho))\|_{T, \gamma} \leq r \sup _{t \in[0, T]} f(t)
$$

where

$$
\begin{aligned}
f(t)= & e^{-(\gamma+1)\left\langle c_{2}\right\rangle t}\left[1+\frac{3\left\langle c_{1}\right\rangle r}{2(2 \gamma+1)\left\langle c_{2}\right\rangle}\left(e^{(2 \gamma+1)\left\langle c_{2}\right\rangle t}-1\right)\right. \\
& \left.+\frac{2}{\gamma+1}\left(e^{(\gamma+1)\left\langle c_{2}\right\rangle t}-1\right)\right] .
\end{aligned}
$$

Note that $f(0)=1$. Additionally

$$
\begin{aligned}
f^{\prime}(t)= & -(\gamma+1)\left\langle c_{2}\right\rangle e^{-(\gamma+1)\left\langle c_{2}\right\rangle t}\left[1+\frac{3\left\langle c_{1}\right\rangle r}{2(2 \gamma+1)\left\langle c_{2}\right\rangle}\left(e^{(2 \gamma+1)\left\langle c_{2}\right\rangle t}-1\right)\right. \\
& \left.+\frac{2}{(\gamma+1)}\left(e^{(\gamma+1)\left\langle c_{2}\right\rangle t}-1\right)\right] \\
& +e^{-(\gamma+1)\left\langle c_{2}\right\rangle t}\left[\frac{3\left\langle c_{1}\right\rangle r}{2} e^{(2 \gamma+1)\left\langle c_{2}\right\rangle t}+2\left\langle c_{2}\right\rangle e^{(\gamma+1)\left\langle c_{2}\right\rangle t}\right]
\end{aligned}
$$

and hence

$$
f^{\prime}(0)=-(\gamma+1)\left\langle c_{2}\right\rangle+\left(\frac{3}{2}\left\langle c_{1}\right\rangle r+2\left\langle c_{2}\right\rangle\right)
$$

Choosing $\gamma>1+\frac{3\left\langle c_{1}\right\rangle r}{2\left\langle c_{2}\right\rangle}$, we have $f^{\prime}(0)<0$, which guarantees existence of $\tilde{T}$ such that $\sup _{t \in[0, \tilde{T}]} f(t)=1$. Taking $T=T^{*}$ for $T^{*} \in[0, \tilde{T}]$ yields

$$
\|F(\rho)\|_{T^{*}, \gamma} \leq r .
$$

Therefore $F(\rho) \in B_{T^{*}, \gamma}(r)$ for $\rho \in B_{T^{*}, \gamma}(r)$. 
Proof of Lemma 4.3. We have

$$
\begin{aligned}
(F(\rho) & -F(\psi))_{t}(x)=\rho_{0}(x) \exp \left(-\int_{0}^{t} h\left(\rho_{s}, x\right) d s\right) \\
& +\int_{0}^{t} R_{2}\left(\rho_{s}, x\right) \exp \left(-\int_{s}^{t} h\left(\rho_{\sigma}, x\right) d \sigma\right) d s \\
& -\rho_{0}(x) \exp \left(-\int_{0}^{t} h\left(\psi_{s}, x\right) d s\right) \\
& -\int_{0}^{t} R_{2}\left(\psi_{s}, x\right) \exp \left(-\int_{s}^{t} h\left(\psi_{\sigma}, x\right) d \sigma\right) d s \\
& =D_{1}+\int_{0}^{t} D_{2} d s,
\end{aligned}
$$

where

$$
D_{1}=\rho_{0}(x)\left[\exp \left(-\int_{0}^{t} h\left(\rho_{s}, x\right) d s\right)-\exp \left(-\int_{0}^{t} h\left(\psi_{s}, x\right) d s\right)\right]
$$

and

$$
\begin{aligned}
D_{2}= & \int_{0}^{t}\left[R_{2}\left(\rho_{s}, x\right) \exp \left(-\int_{s}^{t} h\left(\rho_{\sigma}, x\right) d \sigma\right)\right. \\
& \left.-R_{2}\left(\psi_{s}, x\right) \exp \left(-\int_{s}^{t} h\left(\psi_{\sigma}, x\right) d \sigma\right)\right] d s .
\end{aligned}
$$

Take an arbitrary $T^{*} \in[0, \tilde{T}]$. We have

$$
\left|D_{1}\right| \leq\left\|\rho_{0}\right\|_{L^{\infty}}\left\langle c_{1}\right\rangle \int_{0}^{t}\left\|\rho_{s}-\psi_{s}\right\|_{L^{\infty}} d s \leq r\left\langle c_{1}\right\rangle t e^{\gamma\left\langle c_{2}\right\rangle t}\|\rho-\psi\|_{T^{*}, \gamma} .
$$

To estimate $\left|D_{2}\right|$, consider two cases. First, suppose

$$
\int_{s}^{t}\left(h\left(\rho_{\sigma}, x\right)-h\left(\psi_{\sigma} x\right)\right) d \sigma \geq 0
$$


Then

$$
\begin{aligned}
\left|D_{2}\right| \leq & \mid R_{2}\left(\rho_{s}, x\right) \exp \left[-\int_{s}^{t}\left(h\left(\rho_{\sigma}, x\right)-h\left(\psi_{\sigma}, x\right)\right) d \sigma\right] \\
& -R_{2}\left(\psi_{s}, x\right) \exp \left[-\int_{s}^{t}\left(h\left(\rho_{\sigma}, x\right)-h\left(\psi_{\sigma}, x\right)\right) d \sigma\right] \mid \\
& +\left|R_{2}\left(\psi_{s}, x\right) \exp \left[-\int_{s}^{t}\left(h\left(\rho_{\sigma}, x\right)-h\left(\psi_{\sigma}, x\right)\right) d \sigma\right]-R_{2}\left(\psi_{s}, x\right)\right| \\
\leq & \left|R_{2}\left(\rho_{s}, x\right)-R_{2}\left(\psi_{s}, x\right)\right| \\
& +R_{2}\left(\psi_{s}, x\right)\left\{1-\exp \left[-\int_{s}^{t}\left(h\left(\rho_{\sigma}, x\right)-h\left(\psi_{\sigma}, x\right)\right) d \sigma\right]\right\} .
\end{aligned}
$$

In the other case, when $\int_{s}^{t}\left(h\left(\rho_{\sigma}, x\right)-h\left(\psi_{\sigma}, x\right)\right) d \sigma<0$, we have analogously

$$
\begin{aligned}
\left|D_{2}\right| \leq & \left|R_{2}\left(\rho_{s}, x\right)-R_{2}\left(\psi_{s}, x\right)\right| \\
& \quad+R_{2}\left(\rho_{s}, x\right)\left\{1-\exp \left[-\int_{s}^{t}\left(h\left(\psi_{\sigma}, x\right)-h\left(\rho_{\sigma}, x\right)\right) d \sigma\right]\right\} .
\end{aligned}
$$

Note that both $R_{2}\left(\rho_{s}, x\right)$ and $R_{2}\left(\psi_{s}, x\right)$, as both belong to $B_{T^{*}, \gamma}(r)$, satisfy the same estimate (cf. (4.7))

$$
R_{2}\left(\rho_{s}, x\right), R_{2}\left(\psi_{s}, x\right) \leq \frac{3}{2}\left\langle c_{1}\right\rangle e^{2 \gamma\left\langle c_{2}\right\rangle s} r^{2}+2\left\langle c_{2}\right\rangle e^{\gamma\left\langle c_{2}\right\rangle s} r
$$

which allows us to write

$$
\begin{aligned}
\left|D_{2}\right| \leq & \left|R_{2}\left(\rho_{s}, x\right)-R_{2}\left(\psi_{s}, x\right)\right|+\left(\frac{3}{2}\left\langle c_{1}\right\rangle e^{2 \gamma\left\langle c_{2}\right\rangle s} r^{2}+2\left\langle c_{2}\right\rangle e^{\gamma\left\langle c_{2}\right\rangle s} r\right) \\
& \times\left\{1-\exp \left[-\int_{s}^{t}\left|h\left(\psi_{\sigma}, x\right)-h\left(\rho_{\sigma}, x\right)\right| d \sigma\right]\right\} .
\end{aligned}
$$

We have

$$
\begin{aligned}
& 1-\exp \left[-\int_{s}^{t}\left|h\left(\psi_{\sigma}, x\right)-h\left(\rho_{\sigma}, x\right)\right| d \sigma\right] \leq \int_{s}^{t}\left|h\left(\psi_{\sigma}, x\right)-h\left(\rho_{\sigma}, x\right)\right| d \sigma \\
& =\frac{1}{2} \int_{s}^{t}\left|\int_{\left(\mathbb{R}^{d}\right)^{2}}\left(c_{1}(x, y ; z)+c_{1}(y, x ; z)\right)\left(\rho_{\sigma}(y)-\psi_{\sigma}(y)\right) d y d z\right| d \sigma
\end{aligned}
$$




$$
\begin{aligned}
& \leq \int_{s}^{t}\left\langle c_{1}\right\rangle\left\|\rho_{\sigma}-\psi_{\sigma}\right\|_{L^{\infty}} d \sigma \leq \int_{s}^{t}\left\langle c_{1}\right\rangle e^{\gamma\left\langle c_{2}\right\rangle \sigma}\|\rho-\psi\|_{T^{*}, \gamma} d \sigma \\
& \leq\left\langle c_{1}\right\rangle e^{\gamma\left\langle c_{2}\right\rangle t}(t-s)\|\rho-\psi\|_{T^{*}, \gamma},
\end{aligned}
$$

which yields

$$
1-\exp \left[-\int_{s}^{t}\left|h\left(\psi_{\sigma}, x\right)-h\left(\rho_{\sigma}, x\right)\right| d \sigma\right] \leq\left\langle c_{1}\right\rangle t e^{\gamma\left\langle c_{2}\right\rangle t}|| \rho-\psi \|_{T^{*}, \gamma}
$$

Let us estimate

$$
\begin{aligned}
& \left|R_{2}\left(\rho_{s}, x\right)-R_{2}\left(\psi_{s}, x\right)\right| \\
& \leq \frac{1}{2} \int_{\left(\mathbb{R}^{d}\right)^{2}} c_{1}(y, z ; x) \mid \exp \left(-\int_{\mathbb{R}^{d}} \phi_{1}(x-u) \rho_{s}(u) d u\right) \rho_{s}(y) \rho_{s}(z) \\
& \quad-\exp \left(-\int_{\mathbb{R}^{d}} \phi_{1}(x-u) \psi_{s}(u) d u\right) \psi_{s}(y) \psi_{s}(z) \mid d y d z \\
& +\frac{1}{2} \int_{\left(\mathbb{R}^{d}\right)^{2}}\left(c_{1}(x, y ; z)+c_{1}(y, x ; z)\right) \mid\left[1-\exp \left(-\int_{\mathbb{R}^{d}} \phi_{1}(z-u) \rho_{s}(u) d u\right)\right] \\
& \quad \times \rho_{s}(x) \rho_{s}(y)-\left[1-\exp \left(-\int_{\mathbb{R}^{d}} \phi_{1}(z-u) \psi_{s}(u) d u\right)\right] \psi_{s}(x) \psi_{s}(y) \mid d y d z \\
& +\int_{\mathbb{R}^{d}} c_{2}(y ; x) \mid \exp \left(-\int_{\mathbb{R}^{d}} \phi_{2}(x-u) \rho_{s}(u) d u\right) \rho_{s}(y) \\
& \quad-\exp \left(-\int_{\mathbb{R}^{d}} \phi_{2}(x-u) \psi_{s}(u) d u\right) \psi_{s}(y) \mid d y \\
& +\int_{\mathbb{R}^{d}} c_{2}(x ; y) \mid\left[1-\exp \left(-\int_{\mathbb{R}^{d}} \phi_{2}(y-u) \rho_{s}(u) d u\right)\right] \rho_{s}(x) \\
& \quad-\left[1-\exp \left(-\int_{\mathbb{R}^{d}} \phi_{2}(y-u) \psi_{s}(u) d u\right)\right] \psi_{s}(x) \mid d y .
\end{aligned}
$$

Denote by $I_{i}$ the $i$-th component of the RHS of the above inequality for $i=1,2,3,4$. Then estimating analogously as above, we derive

$$
I_{3}, I_{4} \leq\left\langle c_{2}\right\rangle\left(e^{2 \gamma\left\langle c_{2}\right\rangle s}\left\langle\phi_{2}\right\rangle r+e^{\gamma\left\langle c_{2}\right\rangle s}\right)\|\rho-\psi\|_{T^{*}, \gamma} .
$$


Moreover, noting that

$$
\begin{aligned}
& \left|\rho_{s}(y) \rho_{s}(z)-\psi_{s}(y) \psi_{s}(z)\right| \\
& \leq \frac{1}{2}\left(\rho_{s}(z)+\psi_{s}(z)\right)\left|\rho_{s}(y)-\psi_{s}(y)\right|+\frac{1}{2}\left(\rho_{s}(y)+\psi_{s}(y)\right)\left|\rho_{s}(z)-\psi_{s}(z)\right|,
\end{aligned}
$$

we obtain

$$
\begin{aligned}
& I_{1} \leq \frac{1}{2}\left\langle c_{1}\right\rangle\left(2 e^{2 \gamma\left\langle c_{2}\right\rangle s} r+e^{3 \gamma\left\langle c_{2}\right\rangle s}\left\langle\phi_{1}\right\rangle r^{2}\right)\|\rho-\psi\|_{T^{*}, \gamma} \\
& I_{2} \leq\left\langle c_{1}\right\rangle\left(2 e^{2 \gamma\left\langle c_{2}\right\rangle s} r+e^{3 \gamma\left\langle c_{2}\right\rangle s}\left\langle\phi_{1}\right\rangle r^{2}\right)\|\rho-\psi\|_{T^{*}, \gamma} .
\end{aligned}
$$

Therefore,

$$
\begin{aligned}
\mid R_{2}\left(\rho_{s}, x\right) & -R_{2}\left(\psi_{s}, x\right) \mid \leq\left[\frac{3}{2}\left\langle c_{1}\right\rangle e^{\gamma\left\langle c_{2}\right\rangle s}\left(2 e^{\gamma\left\langle c_{2}\right\rangle s} r+e^{2 \gamma\left\langle c_{2}\right\rangle s}\left\langle\phi_{1}\right\rangle r^{2}\right)\right. \\
& \left.+2\left\langle c_{2}\right\rangle e^{\gamma\left\langle c_{2}\right\rangle s}\left(e^{\gamma\left\langle c_{2}\right\rangle s}\left\langle\phi_{2}\right\rangle r+1\right)\right]\|\rho-\psi\|_{T^{*}, \gamma}
\end{aligned}
$$

Substituting (4.11) and (4.12) into (4.10) and using it together with (4.9), we obtain (cf. (4.8))

$$
\left|(F(\rho)-F(\psi))_{t}(x)\right| \leq e^{\gamma\left\langle c_{2}\right\rangle t} f(t)|| \rho-\psi||_{T^{*}, \gamma},
$$

where

$$
\begin{aligned}
f(t)= & t\left[\frac{3}{2} r^{2}\left\langle c_{1}\right\rangle e^{2 \gamma\left\langle c_{2}\right\rangle t}\left(\left\langle c_{1}\right\rangle t+\left\langle\phi_{1}\right\rangle\right)\right. \\
& \left.+r e^{\gamma\left\langle c_{2}\right\rangle t}\left(2\left\langle c_{1}\right\rangle\left\langle c_{2}\right\rangle t+3\left\langle c_{1}\right\rangle+2\left\langle c_{2}\right\rangle\left\langle\phi_{2}\right\rangle\right)+2\left\langle c_{2}\right\rangle\right] .
\end{aligned}
$$

Therefore,

$$
\|F(\rho)-F(\psi)\|_{T^{*}, \gamma} \leq \sup _{t \in\left[0, T^{*}\right]} f(t)\|\rho-\psi\|_{T^{*}, \gamma} .
$$

Note that $f(t)$ is continuous, increasing function of $t$ and $f(0)=0$. Thus, there exists $T^{* *}>0$ such that $f\left(T^{* *}\right)<1$ and $f(t) \in\left[0, f\left(T^{* *}\right)\right]$ for $t \in$ $\left[0, T^{* *}\right]$. Choosing $T^{*}=\min \left(T^{* *}, \tilde{T}\right)$, we obtain

$$
\|F(\rho)-F(\psi)\|_{T^{*}, \gamma} \leq C\|\rho-\psi\|_{T^{*}, \gamma}
$$

with $C=f\left(T^{*}\right) \leq f\left(T^{* *}\right)<1$.

Acknowledgement. This work has been performed in the framework of the joint Polish-German project No 57154469 "Dynamics of Large Systems of Interacting Entities" supported by DAAD. The author gratefully acknowledges the support and the hospitality extended to him during the stay at Bielefeld University where the main part of the work was done. Special thanks the author wishes to express to Professor Yuri Kozitsky for suggesting the problem and for many fruitful conversations, as without his 
help this paper would not come into being. Finally, the author wishes to express his gratitude to the referee for valuable remarks.

\section{REFERENCES}

[1] Aldous, D. J., Deterministic and stochastic models for coalescence (aggregation and coagulation): a review of the mean-field theory for probabilists, Bernoulli 5 (1) (1999), $3-48$.

[2] Banasiak, J., Kinetic models in natural sciences, in Evolutionary Equations with Applications in Natural Sciences, volume 2126 of Lecture Notes in Math., 133-198, Springer, Cham, 2015.

[3] Banasiak, J., Lamb, W., Langer, M., Strong fragmentation and coagulation with power-law rates, J. Engrg. Math. 82 (2013), 199-215.

[4] Belavkin, V. P., Kolokoltsov, V. N., On a general kinetic equation for many-particle systems with interaction, fragmentation and coagulation, R. Soc. Lond. Proc. Ser. A Math. Phys. Eng. Sci. 459 (2031) (2003), 727-748.

[5] Berns, C., Kondratiev, Y., Kozitsky, Y., Kutoviy, O., Kawasaki dynamics in continuum: micro- and mesoscopic descriptions, J. Dynam. Differential Equations 25 (4) (2013), 1027-1056.

[6] Capitán, J. A., Delius, G. W., Scale-invariant model of marine population dynamics, Phys. Rev. E (3) 81 (6) (2010), 061901, 15pp.

[7] Finkelshtein, D., Kondratiev, Y., Kozitsky, Y., Kutoviy, O., The statistical dynamics of a spatial logistic model and the related kinetic equation, Math. Models Methods Appl. Sci. 25 (2) (2015), 343-370.

[8] Finkelshtein, D., Kondratiev, Y., Kutoviy, O., Vlasov scaling for stochastic dynamics of continuous systems, J. Stat. Phys. 141 (1) (2010), 158-178.

[9] Finkelshtein, D., Kondratiev, Y., Kutoviy, O., Statistical dynamics of continuous systems: perturbative and approximative approaches, Arabian Journal of Mathematics 4 (4) (2015), 255-300.

[10] Finkelshtein, D., Kondratiev, Y., João Oliveira, M., Markov evolutions and hierarchical equations in the continuum. I. One-component systems, J. Evol. Equ. 9 (2) (2009), 197-233.

[11] Kolokoltsov, V. N., Hydrodynamic limit of coagulation-fragmentation type models of k-nary interacting particles, J. Statist. Phys. 115 (5-6) (2004), 1621-1653.

[12] Kondratiev, Y., Kuna, T., Harmonic analysis on configuration space. I. General theory, Infin. Dimens. Anal. Quantum Probab. Relat. Top. 5(2) (2002), 201-233.

[13] Lachowicz, M., Laurençot, P., Wrzosek, D., On the Oort-Hulst-Safronov coagulation equation and its relation to the Smoluchowski equation, SIAM J. Math. Anal. 34 (6) (2003), 1399-1421 (electronic).

[14] Lamb, W., Applying functional analytic techniques to evolution equations, in Evolutionary Equations with Applications in Natural Sciences, volume 2126 of Lecture Notes in Math., 1-46, Springer, Cham, 2015.

[15] Rudnicki, R., Wieczorek, R., Fragmentation-coagulation models of phytoplankton, Bull. Pol. Acad. Sci. Math. 54 (2) (2006), 175-191.

[16] Rudnicki, R., Wieczorek, R., Phytoplankton dynamics: from the behaviour of cells to a transport equation, Math. Model. Nat. Phenom. 1 (1) (2006), 83-100. 
Krzysztof Pilorz

Institute of Mathematics

Maria Curie-Skłodowska University

pl. M. Curie-Skłodowskiej 1

20-031 Lublin

Poland

e-mail: kpilorz@gmail.com

Received August 10, 2015 